\title{
Supply Chain Quality Control Model under Symmetric Information
}

\author{
Haibin Kang, Peiyu Zhu *, Jinling Sun and Hongbin Wang \\ School of Economics and Management, Lanzhou University of Technology, Lanzhou, China. \\ *852646499@qq.com
}

\begin{abstract}
This paper establishes the basic model of supply chain quality control, and discusses the optimal quality effort level under their respective symmetrical information. The main research subjects are risk-neutral supplier and manufacturer. In this paper, the production process of both sides has a common impact on the quality of finished products, and the manufacturing process of the manufacturer does not affect the intermediate products.
\end{abstract}

Keywords: quality control; respective symmetrical information; risk-neutral supplier.

\section{Introduction}

Supply chain quality management (SCQM) combines quality management with supply chain management [1].

Recent years, supply chain quality management has been the focus of supply chain research (He, 2016)[2].It uses advanced management methods and strict control standards to supervise all aspects of product production, so as to ensure that the quality defects of products are controlled within a reasonable range. Because supply chain is dynamic (Grzybowska et al., 2014) [3], the implementation of supply chain quality management mainly depends on the guidance of the core enterprises, which not only need to check their own production process, but also need to develop certain measures to guide the quality of production of other member enterprises (Zhang et al., 2018) [4] to ultimately achieve the overall and individual interests.

\section{Model Formulation}

This chapter will establish the basic model of supply chain quality control and discuss the optimal quality effort level under their respective symmetrical information. Research finds that the wholesale price is also affected by the quality level of products. The main body of the model is a single supplier and a single manufacturer. Supplier manufactures semi-finished products, and the semi-finished products have a certain defect rate. After testing, the manufacturer puts into follow-up production. At this stage, there are four possible results. In reality, there will be testing errors due to testing methods and other reasons. Therefore, this paper assumes that the manufacturer has the possibility of discarding the truth.

\subsection{Basic Assumptions}

Taking into account the reality, the following basic assumptions will be established in this chapter.

Hypothesis 1: the inspection process does not affect the quality of the product;

Hypothesis 2: quality cost input includes production, transportation and other links.

Hypothesis 3: manufacturers will not find semi-finished products with leak detection during processing.

Hypothesis 4: The quality control and profit of the supply chain are considered not only in the case of unit product but also in the case of product order quantity.

Hypothesis 5: influenced by various factors, it may lead to its abandonment and authenticity.

Hypothesis 6: Consumers will find quality defects, but because some quality defects do not affect the use or because of the cost of returns and other factors, only some consumers claim from the manufacturer;

Hypothesis 7: under unit demand. 


\subsection{Symbolic Meaning.}

Table 1. Symbols and meanings of variables

\begin{tabular}{|c|c|}
\hline Variable symbol & Symbolic meaning \\
\hline$P_{s}$ & Supplier's level of quality effort (probability of qualified semi manufactured goods) \\
\hline$P_{m}$ & Manufacturer's quality effort level (probability of qualified products) \\
\hline$\eta_{s}$ & Coefficient of supplier quality cost \\
\hline$\eta_{m}$ & Coefficient of manufacturer quality cost \\
\hline$\eta_{1}$ & Cost coefficient of semi-finished products inspection \\
\hline$\theta_{1}$ & Manufacturer's inspection level for semi-finished products \\
\hline$\omega_{s}$ & Manufacturer's final market price \\
\hline$\omega_{m}$ & The cost of the supplier at the quality level of $P_{S}$ \\
\hline$C_{s}$ & The cost of the manufacturer at the quality level of $P_{m}$ \\
\hline$C_{m}$ & Manufacturer's inspection cost of semi-finished products \\
\hline$T_{1}$ & The cost of internal loss caused by not up to standard. \\
\hline$I$ & External cost of loss \\
\hline$C$ & Expected return function of supplier \\
\hline$\prod_{s}$ & Expected return function of manufacturer \\
\hline$\prod_{m}$ & Expected return function of supply chain as a whole \\
\hline$\prod_{t}$ &
\end{tabular}

Note: $P_{S} \subset[0,1] ; P_{m} \subset[0,1]$; The higher the technology level, the smaller the $\eta_{s}$ and $\eta_{m}$, the smaller the supplier's quality cost; The smaller the $\eta_{m}$, the smaller the manufacturer's production cost; $\theta_{1} \subset[0,1]$.

When $P_{S}>0, C_{s}^{\prime}>0, C_{s}^{\prime \prime}>0$, the parameters are described as:

$C_{s}(0)=C_{s}^{\prime}(0)=0$

Wholesale price is set as a linear function of quality.

$$
\omega_{s}=a_{s}+b_{s} P_{s}
$$

Where, $a_{s}$ and $b_{s}$ are the corresponding parameters.

In order for both parties to have incentives to manufacture, the selling price of the manufacturer to the finished product is greater than or equal to the sum of inspection cost, quality production cost and the wholesale price paid, that is, $\omega_{m} \geq \omega_{s}+T_{1}+C_{s}$ and $\omega_{s} \geq C_{m}$.

Existing research (Balachandran and Radhakrishnan, 2005) [25] shows he proportion of external losses is as follows.

$$
c=a\left(1-P_{s}\right)\left(1-\theta_{1}\right)+b\left(1-P_{m}\right) P_{s} \theta_{1}
$$

Where $a \in[0,1], b \in[0,1]$. A denotes the probability that the supplier will bear its own liability, and $b$ denotes the probability that the supplier will bear the quality loss of the manufacturer. 


\section{Model Solution}

When one party's real quality level information cannot be observed, there will be unilateral moral hazard. When the two sides cannot observe the other party's real information, there will be bilateral moral hazard.

\subsection{Suppliers has Unilateral Moral Hazard}

When supplier has unilateral moral hazard, the supplier's real quality level information cannot be obtained, and the manufacturer's information is true and public. The basic model is that the supplier bears all internal losses and the manufacturer bears all external losses.

The expected profit function of the supplier and manufacture are:

$$
\begin{aligned}
\Pi_{s}= & \omega_{s}\left[1-P_{s}\left(1-\theta_{1}\right)-\left(1-P_{s}\right) \theta_{1}\right]-I\left[P_{s}\left(1-\theta_{1}\right)+\left(1-P_{s}\right) \theta_{1}\right]-C_{s} \\
\Pi_{m}= & \omega_{m}\left[\left(1-P_{s}\right)\left(1-\theta_{1}\right)+\left(1-P_{m}\right) P_{s} \theta_{1}+P_{m} P_{s} \theta_{1}\right]-\omega_{s}\left[1-\left(1-P_{s}\right) \theta_{1}-P_{s}\left(1-\theta_{1}\right)\right] \\
& -C_{m}-T_{1}-\left[\left(1-P_{s}\right)\left(1-\theta_{1}\right)+\left(1-P_{m}\right) P_{s} \theta_{1}\right] e \\
\Pi_{t}= & \omega_{m}\left[P_{s} \theta_{1}\left(1-P_{m}\right)+\left(1-P_{s}\right)\left(1-\theta_{1}\right)+P_{s} \theta_{1} P_{m}\right]-I\left[P_{s}\left(1-\theta_{1}\right)+\left(1-P_{s}\right) \theta_{1}\right]- \\
& e\left[P_{s} \theta_{1}\left(1-P_{m}\right)+\left(1-P_{s}\right)\left(1-\theta_{1}\right)\right]-\frac{1}{2} \eta_{s} P_{s}^{2}-\frac{1}{2} \eta_{m} P_{m}^{2}-\frac{1}{2} \eta_{1} \theta_{1}^{2}
\end{aligned}
$$

Where $\omega_{m}\left[P_{s} \theta_{1}\left(1-P_{m}\right)+\left(1-P_{s}\right)\left(1-\theta_{1}\right)+P_{s} \theta_{1} P_{m}\right]$ denotes the manufacturer's sales revenue, $I\left[P_{s}\left(1-\theta_{1}\right)+\left(1-P_{s}\right) \theta_{1}\right]$ denotes the internal cost of loss, $e\left[P_{s} \theta_{1}\left(1-P_{m}\right)+\left(1-P_{s}\right)\left(1-\theta_{1}\right)\right]$ denotes the loss caused by selling unqualified products to the market, $\frac{1}{2} \eta_{s} P_{s}^{2}$ denotes the supplier's quality cost, the processing cost is included in the formula as a constant. $\frac{1}{2} \eta_{m} P_{m}^{2}$ denotes the manufacturer's quality cost, $\frac{1}{2} \eta_{1} \theta_{1}^{2}$ denotes the manufacturer's inspection cost for the semi-finished product.

$$
\begin{gathered}
\operatorname{Max} \prod_{m} \\
\text { s.t. }(I R) \prod_{s} \geq R_{s} \\
(I C) P_{s} \in \arg \operatorname{Max}_{P_{s}} \prod_{s}
\end{gathered}
$$

$R_{s}$ is the retained earnings of suppliers. Formula (7) is the solution for suppliers to maximize their personal interests.

$$
\begin{gathered}
P_{m}^{*}=-\frac{2 \theta_{1}\left(I+\omega_{m}-e\right) e\left(\theta_{1}-\frac{1}{2}\right)}{\theta_{1}^{2} e^{2}-\eta_{s} \eta_{m}}, \quad P_{s}^{*}=-\frac{2 \eta_{m}\left(I+\omega_{m}-e\right)\left(\theta_{1}-\frac{1}{2}\right)}{\theta_{1}^{2} e^{2}-\eta_{s} \eta_{m}} \\
P_{s 1}=-\frac{2 \theta_{1} I+2 a_{s} \theta_{1}-b_{s} \theta_{1}-I-a_{s}+b_{s}}{4 b_{s} \theta_{1}-2 b_{s}-\eta_{s}}
\end{gathered}
$$

Difference value of $P_{s 1}$ and $P_{s}^{*}$ is: 


$$
\begin{aligned}
A_{1} & =2 \theta_{1} I+2 a_{s} \theta_{1}-b_{s} \theta_{1}-I-a_{s}+b_{s} \\
A_{2} & =\theta_{1}^{2} e^{2}-\eta_{s} \eta_{m} \\
A_{3} & =2 \eta_{m}\left(I+\omega_{m}-e\right)\left(\theta_{1}-\frac{1}{2}\right) \\
A_{4} & =4 b_{s} \theta_{1}-2 b_{s}-\eta_{s} \\
\Delta P_{s} & =P_{s}^{*}-P_{s 1}=\frac{A_{1} A_{2}-A_{3} A_{4}}{A_{3} A_{2}}
\end{aligned}
$$

\subsection{Quality Decision under Bilateral Moral Hazard}

Both sides of the quality level information are hidden, suppliers and manufacturers are only from the perspective of personal interests, there is a risk of reducing their quality level.

$$
\begin{gathered}
\operatorname{Max}_{m} \\
\text { s.t. }(I R) \prod_{s} \geq R_{s} \\
(I C) P_{m} \in \arg \operatorname{Max}_{P_{m}} \prod_{m} \\
(I C) P_{s} \in \arg \operatorname{Max}_{P_{s}} \prod_{s}
\end{gathered}
$$

Formula (11) indicates that the minimum expected return of the supplier is greater than or equal to its retained return, and Formula (12) and Formula (13) mean that both parties take action from the angle of maximizing their own return.

From the formula (12) and (13), the first order optimal solution is obtained respectively for its quality level:

$$
\theta_{1} P_{s} e-\eta_{m} P_{m}=0
$$

The first best solution to the manufacturer's quality level is obtained by formula (11):

$$
b_{s}\left[1-P_{s}\left(1-\theta_{1}\right)-\left(1-\theta_{1}\right) P_{s}\right]+\left(b_{s} P_{s}+a_{s}\right)\left(2 \theta_{1}-1\right)-\eta_{s} P_{s}-I\left(1-2 \theta_{1}\right)=0
$$

Simultaneous (14) and (15) can get:

$$
\begin{gathered}
P_{m 2}=-\frac{\theta_{1} e\left(2 I \theta_{1}+2 a_{s} \theta_{1}-b_{s} \theta_{1}-I-a_{s}+b_{s}\right)}{\eta_{m}\left(4 b_{s} \theta_{1}-2 b_{s}-\eta_{s}\right)} \\
P_{s 2}=-\frac{2 I \theta_{1}+2 a_{s} \theta_{1}-b_{s} \theta_{1}-I-a_{s}+b_{s}}{4 b_{s} \theta_{1}-2 b_{s}-\eta_{s}}
\end{gathered}
$$

Where $P_{m 2}, P_{s 2}$ denote their best solutions. Consider $\Delta P_{s 2}$ the best quality level difference for the supplier under bilateral moral hazard and symmetric information, then: 


$$
\begin{aligned}
& A_{5}=\eta_{m}\left(4 b_{s} \theta_{1}-2 b_{s}-\eta_{s}\right) \\
& A_{6}=\theta_{1}^{2} e^{2}-\eta_{s} \eta_{m} \\
& A_{7}=2 \eta_{m}\left(I+\omega_{m}-e\right)\left(\theta_{1}-\frac{1}{2}\right) \\
& A_{8}=\theta_{1} e\left(2 I \theta_{1}+2 a_{s} \theta_{1}-b_{s} \theta_{1}-I-a_{s}+b_{s}\right) \\
& \Delta P_{s 2}=P_{s}^{*}-P_{s 2}=\frac{A_{8} A_{6}-A_{7} A_{5}}{A_{5} A_{6}}
\end{aligned}
$$

Consider $\Delta P_{m 2}$ the manufacturer's optimal quality level difference under bilateral moral hazard and symmetric information, then:

$$
\begin{aligned}
& A_{5}=\eta_{m}\left(4 b_{s} \theta_{1}-2 b_{s}-\eta_{s}\right) \\
& A_{6}=\theta_{1}^{2} e^{2}-\eta_{s} \eta_{m} \\
& A_{9}=2 \theta_{1}\left(I+\omega_{m}-e\right) e\left(\theta_{1}-\frac{1}{2}\right) \\
& A_{10}=\theta_{1} \eta_{s}\left(2 I \theta_{1}+2 a_{s} \theta_{1}-b_{s} \theta_{1}-I-a_{s}+b_{s}\right) \\
& \Delta P_{m}=P_{m}^{*}-P_{m 2}=\frac{A_{10} A_{6}-A_{9} A_{5}}{A_{5} A_{6}}
\end{aligned}
$$

When there is bilateral moral hazard, due to the double marginalization, the overall benefit of the supply chain composed of both sides will be lower than the expected profit of the supply chain under symmetrical information, and its benefit will be lost.

\section{Conclusion}

This study establishes the basic model of supply chain quality control, explains the inspection process and the probabilities of quality problems, constructs the expected revenue function of supplier and manufacturer under symmetrical information and the overall expected revenue model, and obtains some results by solving the optimal solution under symmetrical information. The conclusion has certain enlightenment to the actual management.

\section{Acknowledgments}

This work was financially supported by Gansu Philosophy and Social Sciences Fund (Number: YB063), Lanzhou University of Technology Youth Foundation Project (Number: 13-0290), Gansu Soft Science Foundation, Science and Technology Project Plan of Constructions of Urban and rural housing construction in Gansu (JK2018-19).

\section{References}

[1]. Robinson, C. J., \& Malhotra, M. K. Defining the concept of supply chain quality management and its relevance to academic and industrial practice. International Journal of Production Economics, 2005, 96(3), 315-337.W. Strunk Jr., E.B. White, The Elements of Style, third ed., Macmillan, New York, 1979.

[2]. He, Y., Xu, Q., Xu, B., \& Wu, P. Supply chain coordination in quality improvement with reference effects. Journal of the Operational Research Society, 2016, 67(9), 1158-1168. 
[3]. Grzybowska, K., \& Kovács, G. Logistics Process Modelling in Supply Chain-algorithm of coordination in the supply chain-contracting. In International Joint Conference SOCO'14CISIS'14-ICEUTE'14 (pp. 311-320). Springer, Cham.P.G. Clem, M. Rodriguez, J.A. Voigt and C.S. Ashley, U.S. Patent 6,231,666. (2001).

[4]. Zhang, J., Cao, Q., \& He, X. Contract and Product Quality in Platform Selling. European Journal of Operational Research. 2018. 